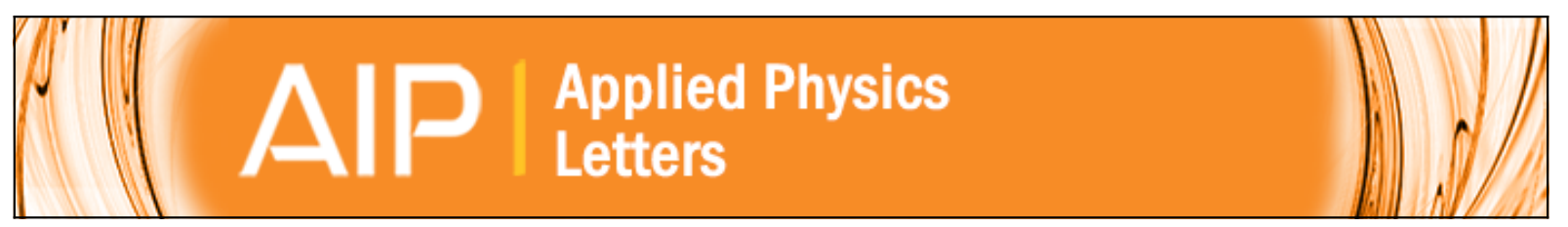

\title{
Polarization self-screening in [0001] oriented InGaN/GaN light-emitting diodes for improving the electron injection efficiency
}

Zi-Hui Zhang, Wei Liu, Zhengang Ju, Swee Tiam Tan, Yun Ji, Xueliang Zhang, Liancheng Wang, Zabu Kyaw, Xiao Wei Sun, and Hilmi Volkan Demir

Citation: Applied Physics Letters 104, 251108 (2014); doi: 10.1063/1.4885421

View online: http://dx.doi.org/10.1063/1.4885421

View Table of Contents: http://scitation.aip.org/content/aip/journal/apl/104/25?ver=pdfcov

Published by the AIP Publishing

\section{Articles you may be interested in}

Origin of InGaN/GaN light-emitting diode efficiency improvements using tunnel-junction-cascaded active regions Appl. Phys. Lett. 104, 051118 (2014); 10.1063/1.4864311

InGaN light-emitting diodes: Efficiency-limiting processes at high injection

J. Vac. Sci. Technol. A 31, 050809 (2013); 10.1116/1.4810789

Improved carrier injection and efficiency droop in InGaN/GaN light-emitting diodes with step-stage multiplequantum-well structure and hole-blocking barriers

Appl. Phys. Lett. 102, 241108 (2013); 10.1063/1.4811735

Origin of InGaN light-emitting diode efficiency improvements using chirped AIGaN multi-quantum barriers Appl. Phys. Lett. 102, 023510 (2013); 10.1063/1.4776739

Hole injection and efficiency droop improvement in InGaN/GaN light-emitting diodes by band-engineered electron blocking layer

Appl. Phys. Lett. 97, 261103 (2010); 10.1063/1.3531753

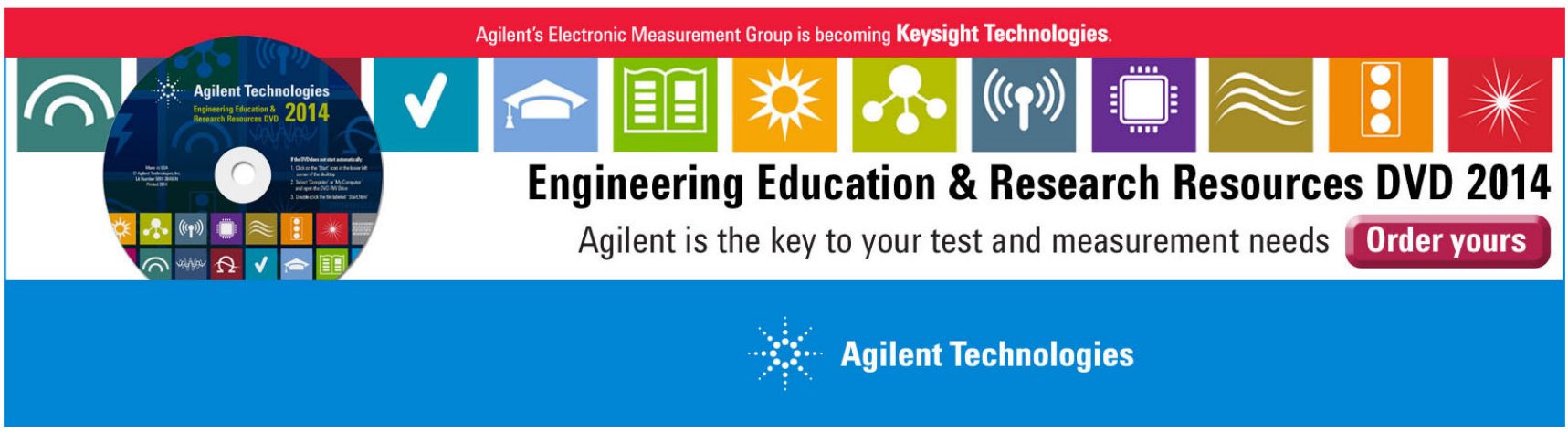




\title{
Polarization self-screening in [0001] oriented InGaN/GaN light-emitting diodes for improving the electron injection efficiency
}

\author{
Zi-Hui Zhang, ${ }^{1}$ Wei Liu, ${ }^{1}$ Zhengang Ju, ${ }^{1}$ Swee Tiam Tan, ${ }^{1}$ Yun $\mathrm{Ji},{ }^{1}$ Xueliang Zhang, ${ }^{1}$ \\ Liancheng Wang, ${ }^{1}$ Zabu Kyaw, ${ }^{1}$ Xiao Wei Sun, ${ }^{1, a)}$ and Hilmi Volkan Demir ${ }^{1,2, a)}$ \\ ${ }^{1}$ LUMINOUS! Centre of Excellence for Semiconductor Lighting and Displays, School of Electrical and \\ Electronic Engineering, School of Physical and Mathematical Sciences, Nanyang Technological University, \\ 50 Nanyang Avenue, Singapore 639798 \\ ${ }^{2}$ Department of Electrical and Electronics, Department of Physics, and UNAM-Institute of Material Science \\ and Nanotechnology, Bilkent University, TR-06800 Ankara, Turkey
}

(Received 28 April 2014; accepted 16 June 2014; published online 24 June 2014)

\begin{abstract}
InGaN/GaN light-emitting diodes (LEDs) grown along the [0001] orientation inherit very strong polarization induced electric fields. This results in a reduced effective conduction band barrier height for the p-type AlGaN electron blocking layer (EBL) and makes the electron blocking effect relatively ineffective and the electron injection efficiency drops. Here, we show the concept of polarization self-screening for improving the electron injection efficiency. In this work, the proposed polarization self-screening effect was studied and proven through growing a p-type EBL with AIN composition partially graded along the [0001] orientation, which induces the bulk polarization charges. These bulk polarization charges are utilized to effectively self-screen the positive polarization induced interface charges located at the interface between the EBL and the last quantum barrier when designed properly. Using this approach, the electron leakage is suppressed and the LED performance is enhanced significantly. (C) 2014 AIP Publishing LLC.

[http://dx.doi.org/10.1063/1.4885421]
\end{abstract}

III-nitride based light-emitting diodes (LEDs) are considered as the ultimate solid state light sources to replace conventional lighting sources. ${ }^{1}$ Thus, substantial efforts have been devoted to improving the efficiency for InGaN/GaN LEDs. One of the obstacles hindering the LED performance is the electron leakage, which has been regarded one of the root causes of the efficiency droop. Therefore, a p-type $\mathrm{AlGaN}$ electron blocking layer (EBL) is often utilized to prevent the electron overflow. As well known, InGaN/GaN LEDs grown along [0001] orientation possess very strong positive polarization charges at the GaN/p-AlGaN EBL interface, which lowers the effective conduction band barrier height for electrons, thus making the EBL relatively ineffective in confining the electrons. One can consider enhancing the electron injection efficiency by increasing the AlN composition or the thickness of the p-type AlGaN EBL. This approach nevertheless increases the barrier height for holes and leads to a reduced hole injection efficiency. ${ }^{2}$ Therefore, it is quite critical to increase the electron confinement efficiency without sacrificing any hole injection. One effective way to improve the electron confinement efficiency is to eliminate/suppress the polarization mismatch between the p-type EBL and the last GaN quantum barrier. In fact, the polarization matched p-type InAIN EBL has been proven to be very useful in reducing the electron leakage, enhancing the LED performance and reducing the efficiency droop. . $^{3,4}$ However, to grow the high-quality InAlN EBL is very challenging compared to the conventional AlGaN EBL. Alternatively, one can employ the polarization inverted p-type AlGaN EBL to suppress the electron leakage level, ${ }^{5}$ which can be achieved, for example, by bonding the [0001]

${ }^{\text {a)} E l e c t r o n i c ~ a d d r e s s e s: ~ e x w s u n @ n t u . e d u . s g ~ a n d ~ v o l k a n @ s t a n f o r d a l u m n i . o r g ~}$ oriented p-GaN/p-AlGaN heterojunction onto the [0001] oriented InGaN/GaN multiple quantum well (MQW) stack. The approach however has to take the damaged interface during the wafer bonding process into consideration when the damaged interface acts as the carrier sink.

In this work, different from the previous reports, the polarization mismatch between the p-type EBL and the last $\mathrm{GaN}$ quantum barrier is proposed to be alleviated by taking advantage of the polarization self-screening effect. Here, the polarization self-screened EBL is realized through grading the AlN composition in the p-type AlGaN EBL. As a result, the effective conduction barrier height is increased and the electron leakage is significantly suppressed. The devices with the proposed polarization self-screened EBL demonstrate superior optical performance compared to the devices with the conventional EBL.

The concept of the proposed self-screened EBL is delineated in Fig. 1. Figs. 1(a) and 1(b) illustrate the conventional p-type EBL and the proposed p-type polarization selfscreened EBL. As well known, the conduction band barrier height $\left(\Phi_{b}\right.$ : defined as the energy difference between the conduction band edge and the Fermi-level for electrons) between the last quantum barrier (LB) and the p-type EBL is given by $\Phi_{b}=\Delta E_{C}-k T \cdot \ln \left(n_{L B / E B L} / N_{C}\right),{ }^{6}$ where $\Delta E_{C}$ is the conduction band offset between the last quantum barrier and the p-type EBL, $k$ is the Boltzmann constant, $T$ is the carrier temperature, $N_{C}$ is the effective electron density of states, and $n_{L B / E B L}$ is the electron concentration accumulated at the interface of the last quantum barrier and the p-type EBL. Unambiguously, one can increase $\Phi_{b}$ by increasing $\Delta E_{C}$ between the last quantum barrier and the p-type EBL through increasing the level of $\mathrm{Al}$ composition. This approach, however, simultaneously increases the valance 


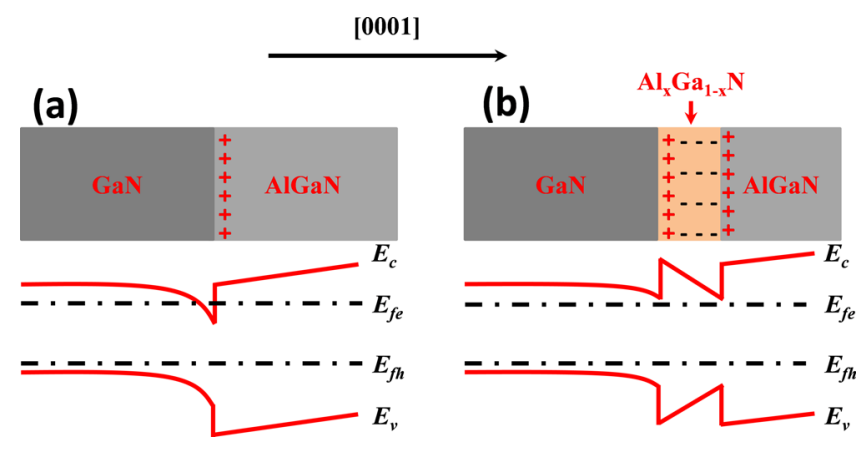

FIG. 1. Schematic drawing for (a) the last GaN quantum barrier and the conventional bulk AlGaN EBL for LED I, and (b) the last GaN quantum barrier and the proposed AlGaN EBL for LED II, along with the respective schematic energy diagrams. $E_{c}, E_{v}, E_{f e}$, and $E_{f h}$ denote the conduction band, valance band, quasi-Fermi level for electrons and quasi-Fermi level for holes, respectively.

band offset and thus retards the hole transport. Another alternative approach for increasing $\Phi_{b}$ can be obtained by reducing the carrier temperature, where the electron thermalization can be realized by using either the InGaN electron cooler ${ }^{7,8}$ or the n-type AlGaN EBL. ${ }^{9}$ The other approach for a larger $\Phi_{b}$ is realized by reducing $n_{L B / E B L}$. According to Fig. 1(a), for the [0001] oriented epitaxial films, the polarization induced positive charges $\left(\sim 1 \times 10^{17} \mathrm{~m}^{-2}\right.$ for the typical $\mathrm{GaN} / \mathrm{Al}_{0.20} \mathrm{Ga}_{0.80} \mathrm{~N}$ heterostructure in the InGaN/GaN LED architecture) at the interface of the last quantum barrier and the p-type AlGaN EBL lead to a very strong electron accumulation, and thus a high $n_{L B / E B L}$ and a severe electron leakage level. Nevertheless, the polarization induced positive charges between the last quantum barrier and the p-type EBL can be partially screened in Fig. 1(b), in which the AIN composition of the p-type $\mathrm{AlGaN}$ is decreasing along the [0001] growth orientation (i.e., $\mathrm{Al}_{\mathrm{x}} \mathrm{Ga}_{1-\mathrm{x}} \mathrm{N}$ ). Here, the polarization induced negative bulk charges are generated in the $\mathrm{Al}_{\mathrm{x}} \mathrm{Ga}_{1-\mathrm{x}} \mathrm{N}$ region. ${ }^{10-15}$ The negative charges are attributed to the compressive strain in the $\mathrm{Al}_{\mathrm{x}} \mathrm{Ga}_{1-\mathrm{x}} \mathrm{N}$ region when the $\mathrm{AlN}$ composition is linearly decreasing along the [0001] orientation. The bulk charge density will be discussed and calculated subsequently. Hence, these charges can partially compensate the positive interface charges. ${ }^{16}$ This results in a reduced electron density at the interface of the last quantum barrier and the p-type EBL, which suppresses $n_{L B / E B L}$ and thus the electron leakage. As a result, enhanced electron injection efficiency, improved optical output power, and reduced efficiency droop are achieved.

To prove the effectiveness of the proposed polarization self-screened p-type EBL structure illustrated in Fig. 1(b) in improving the InGaN/GaN LED performance, two LED epitaxial films (LEDs I and II) have been grown on the c-plane sapphire substrates by a metal-organic chemical vapor deposition (MOCVD) system. The growth was initiated on a $30 \mathrm{~nm}$ thick GaN buffer layer and then followed by an unintentionally n-type $\mathrm{GaN}$ ( $\mathrm{u}-\mathrm{GaN}$ ) layer of $4 \mu \mathrm{m}$ in thickness. After growing the $\mathrm{u}-\mathrm{GaN}$ layer, a $2 \mu \mathrm{m}$ thick $\mathrm{n}-\mathrm{GaN}$ layer doped by $\mathrm{Si}$ dopants was grown serving as the electron source layer with an electron concentration of $5 \times 10^{18} \mathrm{~cm}^{-3}$. Then, the light emitting layers for the two LED samples consist of five-pair $\operatorname{In}_{0.15} \mathrm{Ga}_{0.85} \mathrm{~N} / \mathrm{GaN} \mathrm{MQWs}$, in which the thickness for each quantum well and quantum barrier is set to 3 and $12 \mathrm{~nm}$, respectively. The two samples differ from each other only in the EBL architectures. In LED I, a conventional $20 \mathrm{~nm}$ p-type $\mathrm{Al}_{0.20} \mathrm{Ga}_{0.80} \mathrm{~N}$ EBL was grown. In LED II, the AIN was compositionally graded from $20 \%$ to $0.0 \%$ within the first $10 \mathrm{~nm}$ thickness following a linear profile, which is p-type doped, while the remaining $10 \mathrm{~nm}$ was reserved for the p-type $\mathrm{Al}_{0.20} \mathrm{Ga}_{0.80} \mathrm{~N}$ EBL. Finally, both LED samples were covered by a $0.2 \mu \mathrm{m}$ p-GaN layer. The p-type conductivity was realized through $\mathrm{Mg}$ dopants, and the effective hole concentration in the p-type layer was estimated to be $3 \times 10^{17} \mathrm{~cm}^{-3}$. It should be noted that, the AlGaN layer thickness with grading composition is crucial in producing the three-dimensional hole gas, thus in order to exclude the effect of the three-dimensional hole gas ${ }^{15,17}$ on the enhanced hole injection efficiency and the improved LED performance, we purposely graded the AlN composition within a thin thickness of half of the whole p-type EBL thickness. The electroluminescence (EL) spectra and the optical power output for both LEDs I and II were characterized by a calibrated integrating sphere attached to an Ocean Optics spectrometer (QE65000). The measurements were conducted on the LED dies with a diameter of $1.0 \mathrm{~mm}$ while indium was employed as the metal contacts.

The EL spectra for LEDs I and II are shown in Figs. 2(a) and 2(b) at the current density levels of 10, 20, 30, 40, and $50 \mathrm{~A} / \mathrm{cm}^{2}$, respectively (the EL spectra are collected from the typical emission dies in LEDs I and II). Clearly, we can see that the EL intensity as a function of the injection current density for LED II is stronger than that of LED I. In the
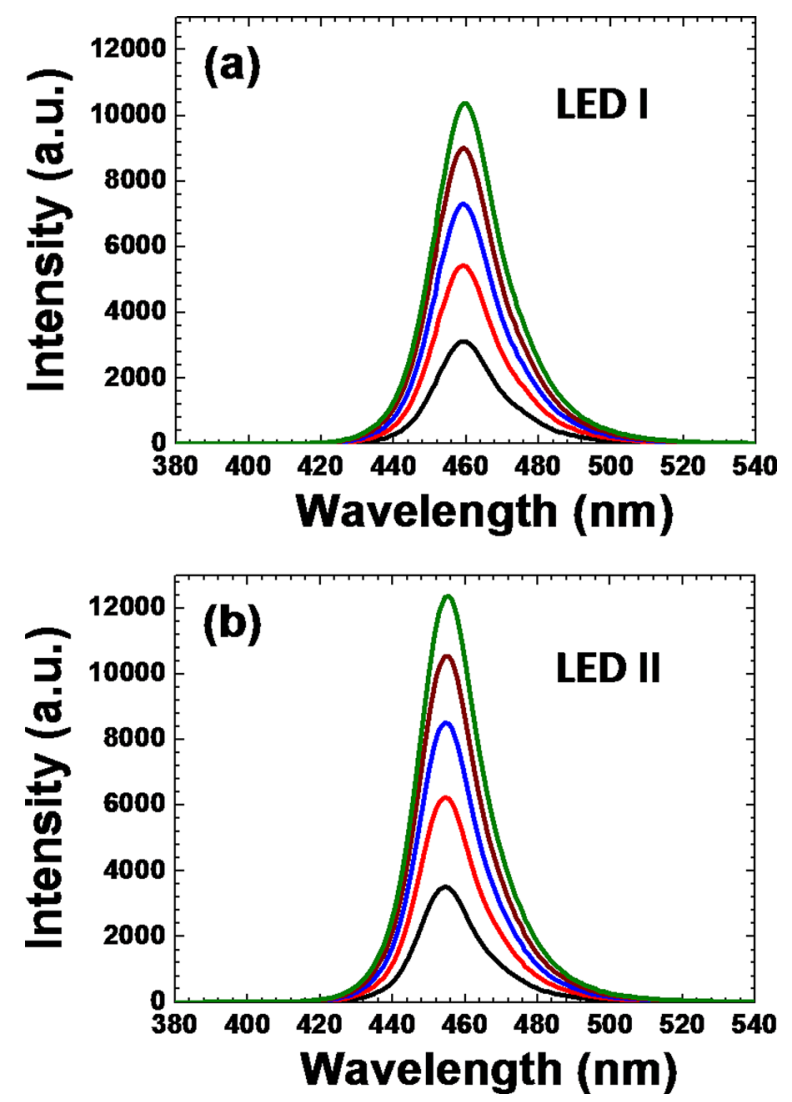

FIG. 2. EL spectra for (a) LED I and (b) LED II at 10, 20, 30, 40, and $50 \mathrm{~A} / \mathrm{cm}^{2}$. 
meanwhile, we also illustrate the integrated optical output power and the external quantum efficiency (EQE) in Fig. 3. Being consistent with the EL profiles in Figs. 2(a) and 2(b), LED II features an enhanced optical output power while maintaining a reduced efficiency droop. For example, the LED II optical power is enhanced by $16.9 \%$ at $100 \mathrm{~A} / \mathrm{cm}^{2}$ when compared to LED I. Furthermore, the efficiency droop at $100 \mathrm{~A} / \mathrm{cm}^{2}$ for LED I and LED II is $49.3 \%$ and $42.3 \%$, respectively. Such an improved performance for LED II is well attributed to the enhanced electron injection efficiency by the suppressed electron leakage level as a result of the proposed p-EBL design. Note that the error bars in Fig. 3 represent the performance variation across the whole epitaxial wafer for LEDs I and II. The performance variation is obtained by calculating the power/EQE difference among the three typical LED dies, each of which is collected at the particular heating zone for the LED wafer mounted on the MOCVD heater during the epitaxial growth.

Additionally, to further understand the physical origin of the aforementioned improved LED performance enabled by the proposed EBL in reducing the electron leakage level, we also performed the numerical simulations using APSYS. The simulation parameters (the energy band offset for any heterojunction, Shockley-Read-Hall (SRH) recombination coefficient, and Auger recombination coefficient) can be found elsewhere. ${ }^{6,8-10}$ More importantly, when calculating the electron capture and escape efficiency, we considered both the ballistic and the quasi-ballistic transports in the InGaN/GaN MQW region. ${ }^{8,9}$ Specifically, the spontaneous and piezoelectric polarizations for the [0001] oriented InGaN/GaN LEDs were included in the numerical simulations, and the polarization effect is represented by setting the polarization charges in the heterojunction regions. The models used to calculate the polarization induced charges were developed by Fiorentini et al. ${ }^{18}$ Moreover, considering the crystal relaxation during the epitaxial process, we assumed a $40 \%$ polarization level. The polarization interface charge density $\left(\sigma_{S}^{p o l}\right)$ between the GaN last barrier and the p$\mathrm{Al}_{0.20} \mathrm{Ga}_{0.80} \mathrm{~N}$ EBL was set to $0.36 \times 10^{17} \mathrm{~m}^{-2}$ while the polarization induced bulk charge density $\left(\rho_{B}^{P o l}\right)$ in the $\mathrm{p}$ $\mathrm{Al}_{\mathrm{x}} \mathrm{Ga}_{1-\mathrm{x}} \mathrm{N}$ region was calculated by

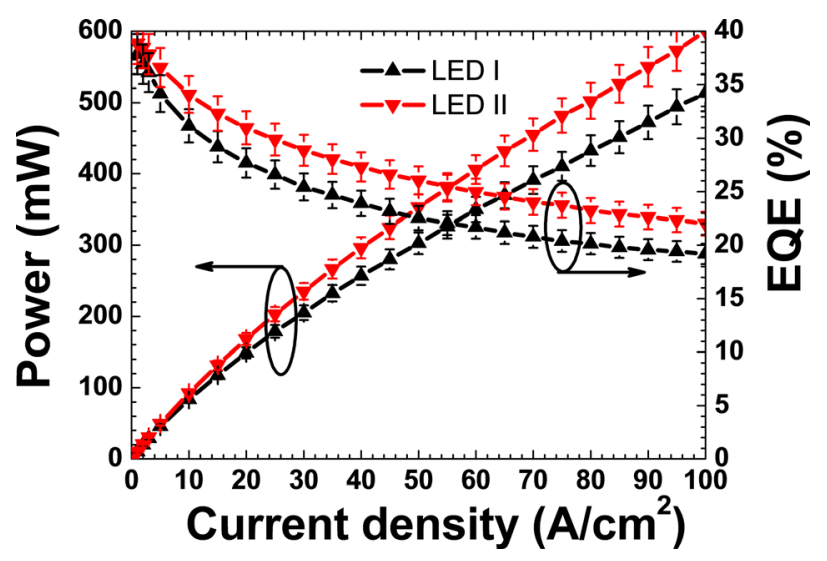

FIG. 3. Experimentally measured optical power and EQE for LEDs I and II. The error bars represent the performance variation across the whole epitaxial wafer, which is obtained by calculating the power/EQE difference among the three typical LED dies collected at different heating zones for the LED wafer.
$\rho_{B}^{P o l}(z)=\nabla \cdot P(z)=(\partial P / \partial x) \times(\partial x / \partial z),{ }^{10}$ where $P(z)$ denotes the polarization density in terms of the grading position $(z) . \rho_{B}^{\text {Pol }}(z)$ of $-3.74 \times 10^{24} \mathrm{~m}^{-3}$ was found. This on one hand, partially compensates $\sigma_{S}^{p o l}$ and, on the other hand, reduces the accumulated electron concentration at the interface of the last quantum barrier and the $\mathrm{p}-\mathrm{Al}_{0.20} \mathrm{Ga}_{0.80} \mathrm{~N}$ EBL. This thus suppresses the electron leakage level across the p-type EBL.

The numerically simulated electron concentration distributions at the current density of $30 \mathrm{~A} / \mathrm{cm}^{2}$ for LEDs I and II around the p-type EBLs and p-GaN regions have been illustrated in Fig. 4. It is demonstrated that the maximum electron density at the interface of the last quantum barrier and the p-type EBL is $\sim 9.97 \times 10^{18} \mathrm{~cm}^{-3}$ and $\sim 2.13 \times 10^{18} \mathrm{~cm}^{-3}$ for LEDs I and II, respectively. The reduced electron density for LED II is well attributed to the screening of $\sigma_{S}^{\text {pol }}$ by $\rho_{B}^{P o l}$ in the $\mathrm{p}-\mathrm{Al}_{\mathrm{x}} \mathrm{Ga}_{1-\mathrm{x}} \mathrm{N}$ region with the $\mathrm{AlN}$ compositional grading. As has been discussed, a reduced electron accumulation at the interface of the last quantum barrier and the p-type EBL is very helpful in increasing the conduction band barrier height $\Phi_{b}$ and thus further alleviating the electron loss, which is also manifested in the electron density in the p-GaN layer for the two LEDs: in LED II the electron density is $\sim 1.46 \times 10^{15} \mathrm{~cm}^{-3}$, much smaller than that of $\sim 1.15 \times 10^{16} \mathrm{~cm}^{-3}$ in LED I. It is worth mentioning that the spike of the electron density at the interface of $\mathrm{p}-\mathrm{Al}_{\mathrm{x}} \mathrm{Ga}_{1-\mathrm{x}} \mathrm{N} /$ $\mathrm{Al}_{0.20} \mathrm{Ga}_{0.80} \mathrm{~N}$ for LED II in Fig. 4 emerges as the twodimensional (2D) electrons are attracted and realigned by the positive polarization induced charges, as shown in Fig. 1(b). The aforementioned polarization self-screening effect in the p-type $\mathrm{Al}_{\mathrm{x}} \mathrm{Ga}_{1-\mathrm{x}} \mathrm{N}$ region significantly reduces the electron accumulation at the interface of the last quantum barrier and the p-type EBL, and this results in a the peak electron density only as low as $\sim 3.39 \times 10^{16} \mathrm{~cm}^{-3}$ at the interface of $\mathrm{Al}_{\mathrm{x}} \mathrm{Ga}_{1-\mathrm{x}} \mathrm{N} / \mathrm{Al}_{0.20} \mathrm{Ga}_{0.80} \mathrm{~N}$ for LED II. Thus, this electron density spike has a little effect to the electron leakage.

Besides showing the electron profiles for LEDs I and II, we also calculated the energy band diagrams at the current density of $30 \mathrm{~A} / \mathrm{cm}^{2}$ in Figs. 5(a) and 5(b). Here, we define $\Phi_{b 1}$ as the conduction band barrier height between the last quantum barrier and the p-EBL, $\Phi_{b 2}$ as the conduction band barrier height of the rest of the p-EBL, $\Phi_{b 3}$ as the valance

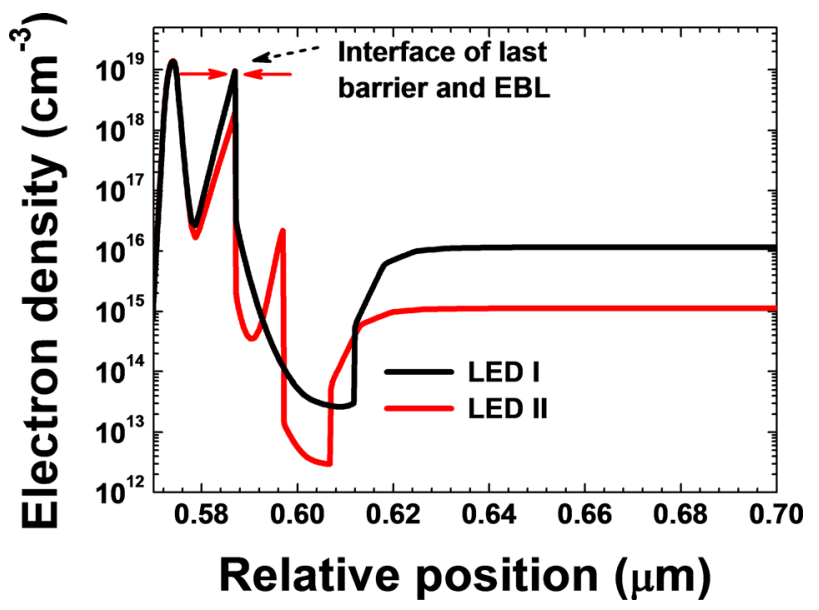

FIG. 4. Simulated electron profile around the EBL region for LEDs I and II at $30 \mathrm{~A} / \mathrm{cm}^{2}$. 


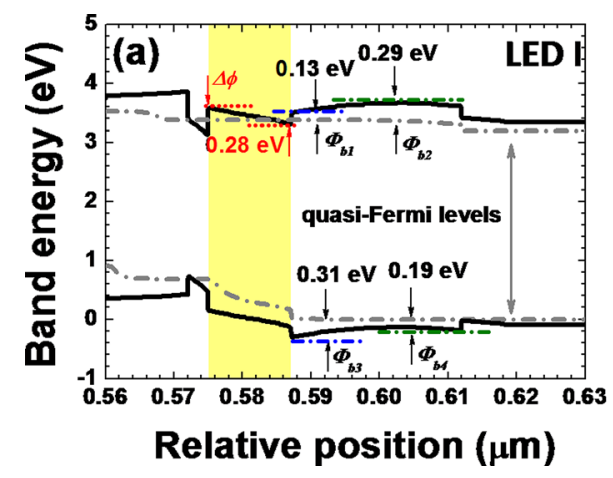

band barrier height between the last quantum barrier and the p-EBL, $\Phi_{b 4}$ as the valance band barrier height of the rest of the p-EBL and $\Delta \phi$ as the energy band bending level of the last quantum barrier. Note that the barrier height is defined as the energy difference between the conduction/valance band and the quasi-Fermi level for electrons/holes. $\Delta \phi$ reflects the energy band bending level of the last quantum barrier, which is partly due to the polarization induced interface charges between the last quantum barrier and the p-type EBL. ${ }^{10}$ The interface charge density has to be reduced for obtaining a small $\Delta \phi$. According to the above discussions, the polarization interface charge density can be partially screened by the polarization induced bulk charges, which can be realized in LED II. For that reason, $\Delta \phi$ has been reduced to $\sim 0.20 \mathrm{eV}$ for LED II from $\sim 0.28 \mathrm{eV}$ for LED I, which makes the last quantum barrier more effective in better confining the electrons in the last quantum well. In addition, a small energy band bending level of the last quantum barrier favors smaller electron accumulation at the interface of the last quantum barrier and the p-EBL. Considering the aforementioned relation $\Phi_{b}=\Delta E_{C}-k T \cdot \ln \left(n_{L B / E B L} / N_{C}\right)$, the conduction band barrier height of $\Phi_{b 1}$ is increased to $\sim 0.21 \mathrm{eV}$ in LED II from $0.13 \mathrm{eV}$ in LED I. Because of the improved electron blocking effect taking place at the interface of the last quantum barrier and the starting position of the p-type EBL, the electron density stored in the rest of the p-EBL can be reduced. This thus leads to an increased $\Phi_{b 2}$ in LED II compared to LED I. Desirably, with this proposed EBL architecture in LED II, the hole injection efficiency seems not affected, which can be read from the values of $\Phi_{b 3} \sim 0.31 \mathrm{eV}$ and $\Phi_{b 4} \sim 0.19 \mathrm{eV}$ in Figs. 5(a) and 5(b) for both LEDs, respectively. Thus, the enhanced optical output power and the reduced efficiency droop observed in LED II are well attributed to the suppressed electron leakage level through the proposed polarization-self screened p-type EBL configuration.

In conclusion, we have demonstrated a concept of reducing the electron leakage level from the multiple quantum well region and improving the light-emitting diode performance by the polarization self-screening effect, which has been realized by employing the p-type AlGaN electron blocking layer with AIN composition partially graded along the [0001] growth orientation. We have achieved an enhanced optical output power and reduced efficiency droop experimentally. With the powerful numerical simulations, we have found a reduced density of the accumulated electrons at the interface of the last quantum barrier and the p-type electron blocking layer stemming from the screening effect on the polarization induced interface positive charges at the interface of the last quantum barrier and the p-type electron blocking layer. For that, the p-type electron blocking layer is very effective in preventing the electrons escaping from the active region of the light-emitting diodes. These results indicate that the concept of polarization selfscreening effect and the proposed electron blocking layer hold great promise for improving the performance of the high-efficiency light-emitting diodes.

This work was supported by the National Research Foundation of Singapore under Grant Nos. NRF-CRP-62010-2, NRF-CRP11-2012-01, and NRF-RF-2009-09 and the Singapore Agency for Science, Technology and Research (A*STAR) SERC under Grant No. 1121202009.

${ }^{1}$ S. T. Tan, X. W. Sun, H. V. Demir, and S. P. DenBaars, IEEE Photon. J. 4, 613-619 (2012).

${ }^{2}$ S.-H. Han, D.-Y. Lee, S.-J. Lee, C.-Y. Cho, M.-K. Kwon, S. P. Lee, D. Y. Noh, D.-J. Kim, Y. C. Kim, and S.-J. Park, Appl. Phys. Lett. 94, 231123 (2009).

${ }^{3}$ S. Choi, H. J. Kim, S.-S. Kim, J. Liu, J. Kim, J.-H. Ryou, R. D. Dupuis, A. M. Fischer, and F. A. Ponce, Appl. Phys. Lett. 96, 221105 (2010).

${ }^{4}$ H. J. Kim, S. Choi, S.-S. Kim, J.-H. Ryou, P. D. Yoder, R. D. Dupuis, A. M. Fischer, K. Sun, and F. A. Ponce, Appl. Phys. Lett. 96, 101102 (2010).

${ }^{5}$ D. S. Meyaard, G.-B. Lin, M. Ma, J. Cho, E. F. Schubert, S.-H. Han, M.-H. Kim, H. Shim, and Y. S. Kim, Appl. Phys. Lett. 103, 201112 (2013).

${ }^{6}$ Z.-H. Zhang, Z. Ju, W. Liu, S. T. Tan, Y. Ji, Z. Kyaw, X. Zhang, N. Hasanov, X. W. Sun, and H. V. Demir, Opt. Lett. 39, 2483-2486 (2014).

${ }^{7}$ V. Avrutin, S. d. A. Hafiz, F. Zhang, Ü. Özgür, H. Morkoç, and A. Matulionis, J. Vac. Sci. Technol. A 31, 050809 (2013).

${ }^{8}$ Z.-H. Zhang, W. Liu, S. T. Tan, Z. Ju, Y. Ji, Z. Kyaw, X. Zhang, N. Hasanov, B. Zhu, S. Lu, Y. Zhang, X. W. Sun, and H. V. Demir, Opt. Express 22, A779-A789 (2014).

${ }^{9}$ Z.-H. Zhang, Y. Ji, W. Liu, S. Tiam Tan, Z. Kyaw, Z. Ju, X. Zhang, N. Hasanov, S. Lu, Y. Zhang, B. Zhu, X. Wei Sun, and H. V. Demir, Appl. Phys. Lett. 104, 073511 (2014).

${ }^{10}$ Z.-H. Zhang, S. Tiam Tan, Z. Kyaw, W. Liu, Y. Ji, Z. Ju, X. Zhang, X. W. Sun, and H. V. Demir, Appl. Phys. Lett. 103, 263501 (2013).

${ }^{11}$ See the supplementary material at http://dx.doi.org/10.1063/1.4885421 on the generation mechanism for the negative polarization induced bulk charges in the $\mathrm{Al}_{\mathrm{x}} \mathrm{Ga}_{1-\mathrm{x}} \mathrm{N}$ region.

${ }^{12}$ L. Zhang, K. Ding, J. C. Yan, J. X. Wang, Y. P. Zeng, T. B. Wei, Y. Y. Li, B. J. Sun, R. F. Duan, and J. M. Li, Appl. Phys. Lett. 97, 062103 (2010).

${ }^{13}$ S. Li, M. Ware, J. Wu, P. Minor, Z. Wang, Z. Wu, Y. Jiang, and G. J. Salamo, Appl. Phys. Lett. 101, 122103 (2012).

${ }^{14} \mathrm{~S}$. Li, T. Zhang, J. Wu, Y. Yang, Z. Wang, Z. Wu, Z. Chen, and Y. Jiang, Appl. Phys. Lett. 102, 062108 (2013).

${ }^{15}$ J. Simon, V. Protasenko, C. Lian, H. Xing, and D. Jena, Science 327, 60-64 (2010).

${ }^{16}$ Z.-H. Zhang, W. Liu, Z. G. Ju, S. T. Tan, Y. Ji, Z. Kyaw, X. L. Zhang, L. Wang, X. W. Sun, and H. V. Demir, Appl. Phys. Lett. 104, 243501 (2014).

${ }^{17}$ L. Zhang, X. C. Wei, N. X. Liu, H. X. Lu, J. P. Zeng, J. X. Wang, Y. P. Zeng, and J. M. Li, Appl. Phys. Lett. 98, 241111 (2011).

${ }^{18}$ V. Fiorentini, F. Bernardini, and O. Ambacher, Appl. Phys. Lett. 80, 1204-1206 (2002). 